\title{
Anchor effects using numerical estimates of simple dot patterns '
}

HARRY HELSON ${ }^{2}$ AND AIKO KOZAKI ${ }^{3}$

YORK UNIVERSITY, Toronto, Ontario

\begin{abstract}
Shifts due to anchor stimuli using absolute judgments have been ascribed to "semantic" and "scale modulus" changes. To test this explanation, random dot patterns of 10,12,14,16 and 18 dots were exposed for 0.30 sec with anchors of 4,13 or 32 dots preceding each of the stimuli. In the control only the series of stimuli were judged. It was found that numerical estimates of the number of dots increased with small anchor, decreased with large anchor, and were not significantly changed with anchor in the vicinity of $A L$. These results exactly parallel those found using methods of absolute and comparative ratings and hence cannot be ascribed to semantic shifts especially in view of the fact that independent groups of $S$ s served in the four anchor and control conditions. Since the anchor effects were significant but not so large that they can be ascribed to change of scale modulus this theory must also be rejected. The AL model seems to offer the simplest and most unitary explanation of these as well as many other results obtained with different types of stimuli and different methods of judging.
\end{abstract}

In many studies concerned with effects of anchors on series stimuli the method of absolute judgment has been used with a categorical response language such as very, very dim to very, very bright or very, very soft to very, very loud. The reliability of such verbal rating scales is attested by highly significant differences between treatment conditions whether different groups or the same Ss are employed across treatments. However, due to the use of verbal categories the criticism has been made that shifts in judgments following introduction of anchors do not represent changes in perceived dimensions of the stimuli but represent only semantic shifts or changes in the modulus of the scale of judgment. While there are very few who today hold to judgmental theories of simultaneous and successive contrast, and while experimental studies have shown that shifts in judgment occur when, as Campbell, Lewis and Hunt say in the title to one of their papers (1958), the judgmental language is absolute, extensive, and extra-experimentally anchored, an experimentum crucis is needed to lay the ghost of the semantic-shift, change-of-modulus theory.

The requirements of such an experimentum crucis seem to consist of the following: first, the stimuli and judgmental task should be as simple as possible to minimize play of verbal factors; second, the response language should be in terms of cardinal numbers learned once and for all; third, the numbers expressing responses should be restricted in range and relatively small so that when shifts occur due to anchors they can not reasonably be ascribed to change of modulus of the judgmental scale. In this study we shall accordingly be concerned with numerical estimates of numbers of dots exposed in random patterns following anchors below, within, and above the "series" stimuli.

\section{Apparatus}

A Harvard type tachistoscope was used for presentation of the stimuli which consisted of random patterns of $10,12,14,16$ or 18 black dots on white background. The dots were 0.25 in. in diameter and were pasted on clear plastic squares 5.0 in. on a side mounted in front of a milk glass screen. The anchor stimuli consisted of random patterns of either 4,13 or 32 dots having the same size as the series dots and similarly mounted. The series stimuli were rotated $90 \mathrm{deg}$ in successive presentations to hinder recognition of the patterns and numbers of dots. The series stimuli were viewed directly by Ss through a half-silvered mirror while the anchor stimuli were seen reflected from the front surface of the mirror. The light sources were two specially-made grids placed behind the milk glass screens containing a mixture of inert gases to provide fairly white, uniform light. They were actuated by $5000-\mathrm{V}$ transformers and had negligible on and off latency. The luminance of the clear plastic areas surrounding both series and anchor dot patterns was adjusted by means of filters to be $2 \mathrm{ft}-\mathrm{L}$ as measured by a Pritchard Spectra photometer. The anchor and series stimuli were exposed for $0.30 \mathrm{sec}$ with $1.0 \mathrm{sec}$ interval between and with the former appearing before each of the latter. All intervals were controlled by means of Hunter interval timers.

\section{Subjects}

Twenty undergraduate students in elementary psychology were randomly divided into four groups of five $S$ s each. In the case of the control group only the series stimuli were judged while in the case of the three experimental or treatment groups either the 4,13 or 32 dot pattern was employed as anchor.

\section{Procedure}

Ss sat in a chair $180 \mathrm{~cm}$ from the stimuli in a dimly-lit room with head resting on a chin rest. Through an opening in the wall they could view the dot patterns exposed in the next room within a black, funnel-like surround. In the center of the stimulus field was a small red dot serving as a fixation point which disappeared when the anchor and series stimuli were flashed. The following instructions were read to each $\mathrm{S}$ :

This is an experiment on estimation of numbers of dots. $A$ number of dots will be exposed briefly in the apparatus in front of you and your task is to judge how many there are. In the center of the field is a red dot to help you fixate. When I say "Ready," look at the red dot until the stimuli appear. I will present two sets of dots successively and you are to judge only the number of dots'in the second. The first set will help you orient to the proper location. You should watch both sets of dots but respond only after the second. Do not try to count the number of dots as there will not be time enough to do so. Make careful estimates and give your judgment as quickly as possible. You will do best by fixating the red dot in the center of the field. Remember, you are to report the number of dots in the second exposure.

Ss were shown all the dot patterns once to acquaint them with the experimental situation after which they were asked to judge the stimuli exposed in random order a total of five times each. The data in this experiment are means of 125 responses ( 5 Ss by 5 stimuli by 5 replications).

\section{RESULTS}

The results are presented in Fig. 1 and show that for each of the stimuli the number of dots reported is highest with the small anchor (4 dot pattern) and smallest with the largest anchor (32 dot pattern). The results for the control and 13-dot anchor groups fall between the other two as expected. In other words, the shifts due to anchors are exactly analogous to the shifts in judgments of lifted weights, loudness, brightness (Helson, 1964) and apparent height of pitches (Rubin, Ware, \& Helson, 1966) using category rating scales. The number of dots is greatly overestimated with the small anchor and underestimated with the large anchor. The estimates by the control group are fairly close to the actual number of dots exposed and are not significantly different from them. Introduction of the 13-dot anchor which is somewhat below average of the series stimuli was for the purpose of determining the adaptation level operative under these conditions of judging. Operationally the number of dots at $\mathrm{AL}$ is that number that, used 


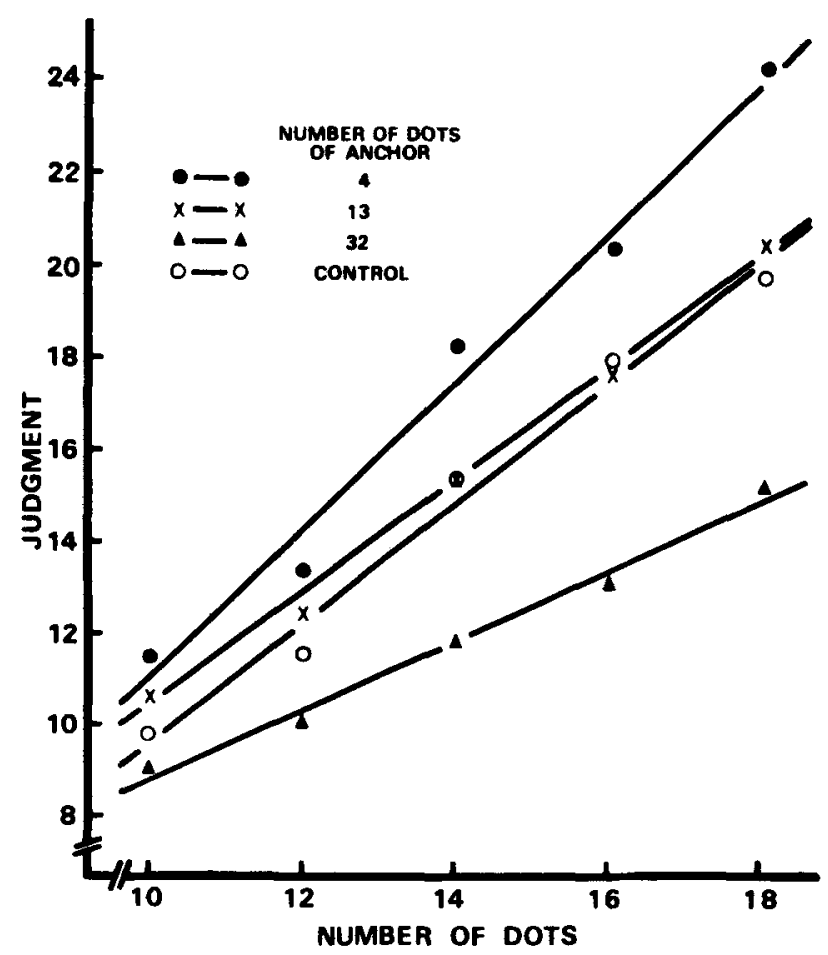

Fig. 1. Numerical estimates of numbers of dots in random patterns by independent groups with anchors of 4,13 and 32 dots and by a control group that judged without any anchor.

as anchor, neither raises nor depresses estimates of the series stimuli. This number used as anchor yielded estimates that are not significantly different from the no-anchor or control condition. It should be noted that AL of 13 is below the geometric mean of the series stimuli. This finding also parallels results using category-type judgments in various sense modalities (Helson, 1964).

While the plotted points and fitted curves in Fig. 1 are clearly separated (excluding the data for 13 anchor which were not expected to be different from the control as pointed out above) and the overall $F$ test for conditions is statistically significant $(F=5.229$, df $=3 / 16, p<.05)$, only two of the conditions are significantly different: the 4 nd 32 anchor conditions $(F=15.456, \mathrm{df}=1 / 16, \mathrm{p}<.01)$ and the 13 and 32 anchor conditions $(F=5.669$, df $=1 / 16, p<.05)$. The most significant source of variance is naturally that due to series stimuli $(F=146.527, \mathrm{df}=4 / 64, \mathrm{p}<.001)$. The significant interaction between anchors and series stimuli $(F=3.280$, df $=12 / 24$, $p<.01)$ shows that anchor conditions differentially affect judgments of series stimuli, a finding that also agrees with results from methods of absolute judgment.

\section{DISCUSSION}

This experiment has shown that the main phenomena found with the methods of absolute and comparative ratings appear when judgments are made in terms of numbers: the number of dots reported shift upward with anchors below the series, downward with anchors above the series, and not significantly with anchor in the vicinity of the prevailing $A L$. The tendency for anchors to cause more downward than upward displacement found in judgments of lightness, weight and loudness with the method of absolute judgment using category ratings is also found with numerical judgments as shown by comparing the curves for 4 and 32 anchor conditions with the control condition in Fig. 1.

It thus appears that when using a simple, objectively oriented task requiring numerical judgments, over- and underestimation with small and large anchors occur just as they do when verbal categories are used for the response language. While the shifts in judgments with anchors are significant they are not so large that they can be attributed to change in modulus of the Ss' scales. In fact the concept of change in modulus is not applicable under these conditions because Ss are not given, or asked to assign, an arbitrary number to any stimuli as is usually done in magnitude estimation (cf., Poulton, 1968). The influence of anchors under our conditions must be exerted at fairly basic levels, as in the case of simultaneous and successive visual contrast, for Ss are instructed to judge only the series stimuli. Furthermore, since different groups judged under each of the anchor and control conditions the concept of "semantic shift" is not applicable because there was no previous set of judgments from which shift could have occurred. Hence we must seek an explanation of the differences between groups within each of the conditions under which Ss judged the stimuli.

We have thus found the main effects of anchors characteristic of absolute judgments of lifted weights, loudness, pitch, lightness, and chromaticness (Helson, 1964; Rubin, Ware, \& Helson, 1966) with numerical judgments. A single explanation embracing category ratings and judgments in terms of cardinal numbers is desirable if the criterion of simplicity has any value in psychological theorizing. "The supreme criterion of scientific theory is simplicity" said the English physicist Whyte (quoted by Kluver, 1958). Certainly the pooling model of AL theory provides a simpler quantitative approach to the anchor and series effects found in psychophysical judgments than do theories that resort to vague references to semantic and other processes. The assumption that series and anchor stimuli pool to form reference levels seems to be the most straightforward and simplest yet made to account for phenomena common to different types of stimuli and different methods of judging them.

\section{REFERENCES}

CAMPBELL, D. T., LEWIS, N. A., \& HUNT, W. A. Context effects with judgmental language that is absolute, extensive, and extraexperimentally anchored. J. exp. Psychol., 1958, 55, 220-228.

HELSON, H. Adaptation-Level Theory. New York: Harper and Row, 1964. KLÜVER, H. Karl S. Lashley. Res. Publ. nerv. men. Dis., 1958, 36, 14-15.

RUBIN, E. D., WARE, M. E., \& HELSON, H. Anchor-effects in pitchlocalization. Amer. J. Psychol, 1966, 79, 458463.

POULTON, E. C. The new psychophysics: Six models for magnitude estimation. Psychol. Bull., 1968, 69, 1-19.

\section{NOTES}

1. This work was supported in part by a grant from the National Research Council of Canada. A study by Donald L. Corson at Kansas State University provided much valuable information regarding choice of the conditions under which the present investigation was made. In view of the work of Corson we can regard this study as a replication and confirmation of his results with different time constants and dot patterns. We are also indebted to Dr. Takashi Kozaki for statistical analyses and other help in preparation of this report.

2. Now at University of Massachusetts.

3. Now at Tokyo Woman's Christian College.

(Accepted for publication May 23, 1968.) 